\title{
Spin evolution of a proto-neutron star
}

\author{
Giovanni Camelio, ${ }^{1, *}$ Leonardo Gualtieri, ${ }^{1, \dagger}$ José A. Pons, ${ }^{2, \downarrow}$ and Valeria Ferrari ${ }^{1, \S}$ \\ ${ }^{1}$ Dipartimento di Fisica, "Sapienza" Università di Roma \& Sezione INFN Romal, \\ P.A. Moro 5, 00185 Roma, Italy \\ ${ }^{2}$ Departament de Física Aplicada, Universitat dAlacant, Ap. Correus 99, 03080 Alacant, Spain
}

(Received 14 January 2016; published 5 July 2016)

\begin{abstract}
We study the evolution of the rotation rate of a proto-neutron star, born in a core-collapse supernova, in the first seconds of its life. During this phase, the star evolution can be described as a sequence of stationary configurations, which we determine by solving the neutrino transport and the stellar structure equations in general relativity. We include in our model the angular momentum loss due to neutrino emission. We find that the requirement of a rotation rate not exceeding the mass-shedding limit at the beginning of the evolution implies a strict bound on the rotation rate at later times. Moreover, assuming that the proto-neutron star is born with a finite ellipticity, we determine the emitted gravitational wave signal and estimate its detectability by present and future ground-based interferometric detectors.
\end{abstract}

DOI: $10.1103 /$ PhysRevD.94.024008

\section{INTRODUCTION}

When a supernova explodes, it leaves a hot, lepton-rich, and (presumably) rapidly rotating remnant: a proto-neutron star (PNS). In the early stages of its evolution, the PNS cools down and loses its high lepton content, while its radius and rotation rate decrease. In this phase, a huge amount of energy and of angular momentum is released, mainly due to neutrino emission [1-3]. A fraction of this energy is expected to be emitted in the gravitational wave channel; indeed, as a consequence of the violent collapse, nonradial oscillations can be excited, making PNSs promising sources for present and future gravitational detectors [4-7].

In the first tenths of seconds after its birth, the PNS is turbulent and characterized by large instabilities. During the next tens of seconds, it undergoes a more quiet, "quasistationary" evolution (the Kelvin-Helmholtz phase), which can be described as a sequence of equilibrium configurations [1,3]. In this article, we study the evolution of the rotation rate of a PNS during this quasistationary, Kelvin-Helmholtz phase. An accurate modeling of this phase is needed, for instance, to compute the frequencies of the PNS gravitational wave emission. Moreover, it provides a link between supernova explosions, a phenomenon which is still not fully understood, and the properties of the observed population of young pulsars. Current models of the evolution of progenitor stars [8], combined with numerical simulations of core collapse and explosion (see, e.g., [9-13]), do not allow sufficiently accurate estimates of the expected rotation rate of newly born PNSs; they only show that the minimum rotation period

\footnotetext{
*giovanni.camelio@roma1.infn.it leonardo.gualtieri@roma1.infn.it jose.pons@ua.es

§valeria.ferrari@roma1.infn.it
}

at the onset of the Kelvin-Helmoltz phase can be as small as a few milliseconds, if the spin rate of the progenitor is sufficiently high. On the other hand, astrophysical observations of young pulsar populations (see [14] and references therein) show typical periods $\gtrsim 100 \mathrm{~ms}$.

The quasistationary evolution of a PNS driven by neutrino transport in a spherically symmetric spacetime has been extensively studied in the past, quite often adopting an equation of state (EOS) obtained within a finite-temperature, field-theoretical model solved at the mean field level [3,15-17]. This approach yields a sequence of thermodynamical profiles describing the structure and the early evolution of a nonrotating PNS. A different approach has been used in [6], where an EOS obtained within a finite-temperature, many-body theory approach was employed, but the neutrino transport equations were not explicitly solved (a set of entropy and lepton fraction profiles were adopted, having the same qualitative behavior as those of [3]). We also mention that the nonradial oscillations of the quasistationary configurations obtained with these different approaches have been studied in $[4,6,18]$, where the quasinormal mode frequencies of the gravitational waves emitted in the early PNS life were computed.

The evolution of rotating PNSs has been studied in [19], where the thermodynamical profiles obtained in [3] for a nonrotating PNS were employed as effective one-parameter EOSs; the rotating configurations were obtained using the nonlinear BGSM code [20] to solve Einstein's equations. A similar approach has been followed in [21], which used the profiles of [3] and [6]. The main limitations of these works are the following:

(i) The evolution of the PNS rotation rate is due not only to the change in the moment of inertia (i.e., to the contraction) but also to the angular momentum change due to neutrino emission [22]. This was 
neglected in [19] and described with an heuristic formula in [21].

(ii) As we discuss in this paper, when the PNS profiles describing a nonrotating star are treated as an effective EOS, one can obtain configurations which are unstable to radial perturbations.

In this article, we study the quasistationary evolution of a spherically symmetric PNS, solving the relativistic equations of neutrino transport and of stellar structure. The details of our code are discussed in [23], where it is applied to more recent EOSs. Here, we employ the same EOS used in [3] (i.e., GM3 [24]) to study the spin evolution of the PNS in its first tens of seconds of life. To model an evolving, rotating PNS, we use the profiles of entropy per baryon and lepton fraction $s(a), Y_{L}(a)$ ( $a$ is the number of baryons enclosed in a sphere passing through the point considered) obtained with our evolution code which describes a nonrotating PNS. Our approach is different from that used in [19], as discussed in detail in Sec. III B. In order to determine the PNS spin evolution, we model the evolution of angular momentum (due to neutrino emission) using Epstein's formula [22]. We also discuss the gravitational wave emission which could be associated with this process.

The plan of the paper is as follows. In Sec. II we briefly describe our approach to model the PNS evolution in its quasistationary phase. In Sec. III we describe our model of a rotating PNS. In Sec. IV we show our results, and in Sec. V we draw our conclusions. The details of the slowly rotating model are described in the Appendix.

\section{EARLY EVOLUTION OF A PROTO-NEUTRON STAR}

The quasistationary, Kelvin-Helmholtz phase of a PNS starts a few hundreds of milliseconds after the core bounce $[1,3]$. This phase consists of two evolutionary stages. In the first few tens of seconds, neutrinos diffuse from the lowentropy core to the high-entropy envelope, deleptonizing the core and increasing its temperature. In the second phase, the star is lepton poor but hot, the entropy gradient is smoothed out, and thermally produced neutrinos cool down the PNS. After about one minute, the star becomes transparent to neutrinos and can be considered as a "mature" neutron star, with a radius of $\sim 10-15 \mathrm{~km}$ and a temperature $<1 \mathrm{MeV}$.

The PNS evolution in the Kelvin-Helmholtz phase can be considered as a sequence of quasistationary configurations because the hydrodynamical time scale is much smaller than the evolution time scale. Following [3], we model this phase by solving the general relativistic neutrino transport equations coupled with the structure equations, assuming spherical symmetry. In each quasistationary configuration, the spacetime metric has the form

$d s^{2}=-e^{\phi(r)} d t^{2}+e^{\lambda(r)} d r^{2}+r^{2}\left(d \theta^{2}+\sin ^{2} \theta d \varphi^{2}\right)$, where $\phi(r)$ and $\lambda(r)$ are radial functions obtained by solving the Tolman-Oppenheimer-Volkov (TOV) equations (in this paper we use geometrized units, in which $c=G=1)$. The perfect fluid of the star is described by the stress-energy tensor $T_{\mu \nu}=(\epsilon+p) u_{\mu} u_{\nu}+p g_{\mu \nu}$, where $u^{\mu}=\left(e^{-\phi / 2}, 0,0,0\right)$ is the fluid four-velocity and $\epsilon, p$ are the energy density and pressure of the fluid, respectively. The gravitational mass inside a radius $r$ is $m(r)=r\left(1-e^{-\lambda}\right) / 2$. At the surface of the star, $r=R$, the pressure vanishes and the metric matches with the exterior Schwarzschild metric, with $M=m(R)$ as the gravitational mass of the star. We also define the baryon number inside a radius $r$,

$$
a(r)=4 \pi \int_{0}^{r} e^{\lambda / 2} n_{b} r^{\prime 2} d r^{\prime},
$$

where $n_{b}$ is the baryon number density. The position inside the star can be described either by the coordinate $r$ or by the enclosed baryon number $a$. We also define the rest-mass density $\rho=m_{n} n_{b}$ ( $m_{n}$ is the neutron mass), and the baryon mass of the star $M_{b}=m_{n} a(R)$. We use $M_{b}=1.60 M_{\odot}$, which corresponds, in the calculations of this paper, to a gravitational mass of $1.55 M_{\odot}$ at $200 \mathrm{~ms}$ from the core bounce, which reduces to $\sim 1.4 M_{\odot}$ in the first ten seconds of life of the PNS.

Since the PNS has a temperature of several MeV, its EOS is nonbarotropic and can be written as

$$
\epsilon=\epsilon\left(p, s,\left\{Y_{i}\right\}_{i}\right),
$$

where $s$ is the entropy per baryon and $Y_{i}=n_{i} / n_{b}$ is the fraction of the $i$ th species, with number density $n_{i}$. Assuming that the matter is in beta equilibrium, the dependence on the composition $\left\{Y_{i}\right\}_{i}$ can be cast into a dependence on only the electron-type lepton fraction $Y_{L}=n_{L} / n_{b}$. Different choices of thermodynamical variables are possible, for instance, replacing the entropy per baryon $s$ with the temperature $T$. In this paper we use the finite-temperature EOS GM3 of Glendenning and Moszkowski [24], obtained within a field-theoretical model solved at the mean field level, where the interactions between baryons are mediated by the exchange of mesons; it contains only nucleonic degrees of freedom. This is the same EOS employed in [3]; we consider the case of matter composed by electrons, protons, and neutrons. More recent EOSs, based on a many-body theory approach, will be considered in a future work [23].

In order to solve the TOV equations, we need to know, at each point, the energy density as a function of the pressure; thus, we need to know the EOS and the thermodynamical profiles $s(a), Y_{L}(a)$, which are obtained by solving the transport equations 


$$
\begin{gathered}
\frac{\partial Y_{L}}{\partial t}+\frac{\partial\left(4 \pi e^{\phi / 2} r^{2} F_{\nu}\right)}{\partial a}=0 \\
T \frac{\partial s}{\partial t}+\mu_{\nu} \frac{\partial Y_{L}}{\partial t}+e^{-\phi / 2} \frac{\partial\left(4 \pi e^{\phi} r^{2} H_{\nu}\right)}{\partial a}=0
\end{gathered}
$$

where $F_{\nu}$ and $H_{\nu}$ are, respectively, the neutrino number and energy fluxes

$F_{\nu}=-\frac{\mathrm{e}^{-\frac{\lambda+\phi}{2}} T^{2}}{6 \pi^{2} \hbar^{3}}\left[D_{3} \frac{\partial\left(T \mathrm{e}^{\phi / 2}\right)}{\partial r}+\left(T \mathrm{e}^{\phi / 2}\right) D_{2} \frac{\partial \eta}{\partial r}\right]$

$H_{\nu}=-\frac{\mathrm{e}^{-\frac{\lambda+\phi}{2}} T^{3}}{6 \pi^{2} \hbar^{3}}\left[D_{4} \frac{\partial\left(T \mathrm{e}^{\phi / 2}\right)}{\partial r}+\left(T \mathrm{e}^{\phi / 2}\right) D_{3} \frac{\partial \eta}{\partial r}\right]$,

where $\eta=\mu_{\nu} / T$ is the electron-type neutrino degeneracy parameter and $D_{n}$ are the neutrino diffusion coefficients, which are computed assuming the diffusion approximation [3]. In order to preserve causality and stabilize the code in the semitransparent regions near the PNS surface, we apply a flux-limiter [25].

Our code evolves the PNS by iteratively solving, at each time step, (i) the transport equations (4) and (5) using an implicit scheme and (ii) the TOV equations by relaxation method. The time evolution keeps the baryon mass $M_{b}$ constant and provides, at each time step, a quasistationary configuration of the (nonrotating) star, described by the profiles of all the thermodynamical quantities $\left(p, \epsilon, n_{b}, s\right.$, $Y_{L}$, etc.) as functions of $a$ (or of $r$ ). We start our integration at $200 \mathrm{~ms}$ from core bounce. The initial profiles (which are the same employed in [3]) are the result of core-collapse simulations [26]. In Figs. 1 and 2 we show the evolutionary profiles of the entropy per baryon $s$ and the electron-type lepton fraction $Y_{L}$ as functions of the enclosed baryon mass. We have checked that the total energy and lepton number are conserved during the evolution within a few

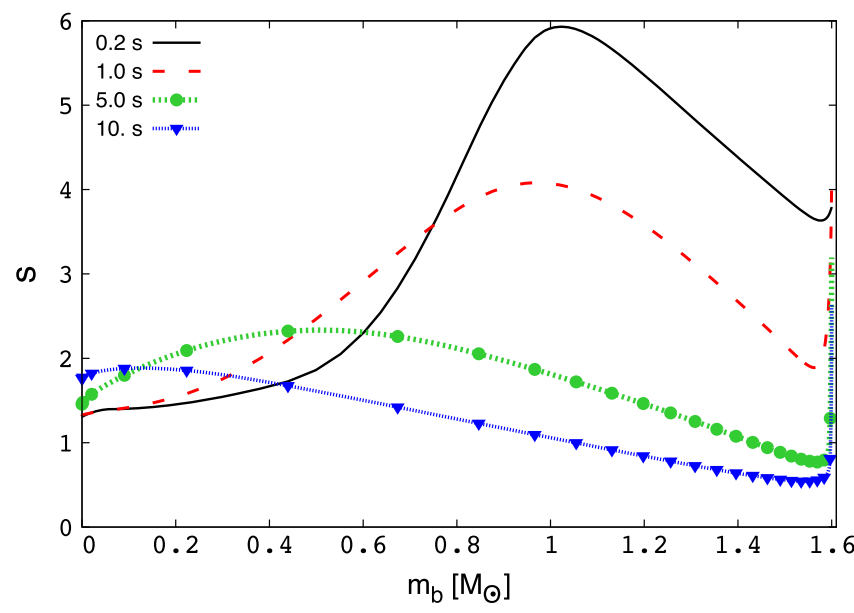

FIG. 1. Entropy per baryon as a function of the enclosed baryon mass $m_{b}=m_{n} a$ at $t=0.2,1,5,10 \mathrm{~s}\left(k_{\mathrm{B}}=1\right)$.

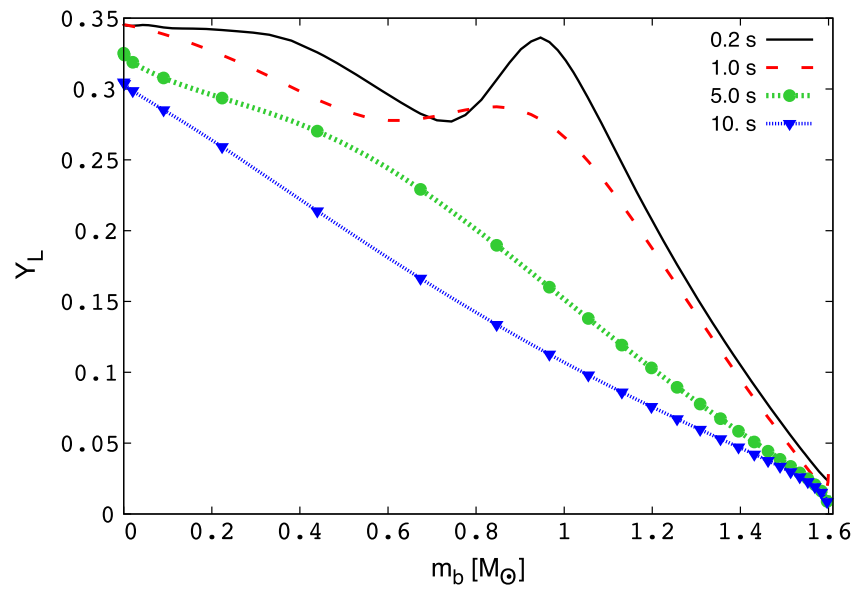

FIG. 2. Electron-type lepton fraction as a function of the enclosed baryon mass at $t=0.2,1,5,10 \mathrm{~s}$.

percent in the early stages of the evolution and with more accuracy in later stages. We remark that this error can be significantly reduced by reducing the time step; however, this accuracy is sufficient for the aims of this work. Our code will be described in detail in a future work [23].

Recently, different approaches have been applied in the study of the PNS evolution (see, e.g., [17]), in which the neutrino spectrum is described with greater accuracy by means of multigroup codes. However, since in this work we are not interested in the details of the neutrino emission, we prefer to employ a simpler and faster energy-averaged approach (as in [3]). As mentioned above, our code also employs a flux-limiter [25], which makes it difficult to establish the precise location of the neutrino sphere. Both the neutrino sphere and the neutrino spectra are better determined with more complex core-collapse codes, which however are far slower, while our PNS code is suitable to run for longer evolution times. Typical core-collapse codes run for at most $500 \mathrm{~ms}$ after core bounce, whereas we can easily explore the first minute of PNS life, at the end of which the star becomes neutrino-transparent.

\section{A MODEL OF ROTATING PROTO-NEUTRON STARS}

\section{A. Slowly rotating stars in general relativity}

We model a rotating PNS using the perturbative approach of Hartle and Thorne [27,28] (see also [29]). The rotating star is described as a stationary perturbation of a spherically symmetric background for small values of the angular velocity $\Omega=2 \pi \nu$, i.e., $\nu \ll \nu_{\mathrm{ms}}$ ( $\nu_{\mathrm{ms}}$ is the massshedding frequency, at which the star starts losing mass at the equator, see Sec. III D). As shown in [21], this "slow rotation" approximation is reasonably accurate for rotation rates up to $\sim 0.8$ of the mass-shedding limit, providing values of mass, equatorial radius, and moment of inertia which differ by $\lesssim 0.5 \%$ from those obtained with fully 
relativistic, nonlinear simulations. In our approach we assume uniform rotation; PNSs are expected to have a significant amount of differential rotation at birth [30], which, however, is likely to be removed by viscous mechanisms, such as, for instance, magnetorotational instability [31], in a fraction of a second.

This work should be considered as a first step towards a more detailed description of rotating PNSs, in which we include differential rotation.

The spacetime metric, up to third order in $\Omega$, can be written as

$$
\begin{aligned}
d s^{2}= & -e^{\phi(r)}\left[1+2 h_{0}(r)+2 h_{2}(r) P_{2}(\mu)\right] d t^{2} \\
& +e^{\lambda(r)}\left[1+\frac{2 m_{0}(r)+2 m_{2}(r) P_{2}(\mu)}{r-2 M(r)}\right] d r^{2} \\
& +r^{2}\left[1+2 k_{2}(r) P_{2}(\mu)\right]\left[d \theta^{2}+\sin ^{2} \theta\{d \phi-[\omega(r)\right. \\
& \left.\left.\left.+w_{1}(r)+w_{3}(r) P_{3}^{\prime}(\mu)\right] d t\right\}^{2}\right],
\end{aligned}
$$

where $\mu=\cos \theta$ and $P_{n}(\mu)$ is the Legendre polynomial of order $n$, the prime denoting the derivative with respect to $\mu$. The perturbations of the nonrotating star are described by the functions $\omega$ [of $O(\Omega)], h_{0}, m_{0}$, and $h_{2}, m_{2}, k_{2}$ [of $O\left(\Omega^{2}\right)$ ], and $w_{1}, w_{3}$ [of $O\left(\Omega^{3}\right)$ ]. The energy-momentum tensor is

$$
T^{\mu \nu}=(\mathcal{E}+\mathcal{P}) u^{\mu} u^{\nu}+\mathcal{P} g^{\mu \nu},
$$

where $g_{\mu \nu}, u^{\mu}$ are the metric and four-velocity in the rotating configuration, and we denote by calligraphic letters thermodynamical quantities (energy, density, and pressure) in the rotating star. An element of fluid, at position $(r, \theta)$ in the nonrotating star, is displaced by rotation to the position

$$
\bar{r}=r+\xi(r, \theta),
$$

where $\xi(r, \theta)=\xi_{0}(r)+\xi_{2}(r) P_{2}(\mu)+O\left(\Omega^{4}\right) \quad$ is the Lagrangian displacement.

In the Hartle-Thorne approach, one assumes that if the fluid element of the nonrotating star has pressure $P$ and energy density $\epsilon$, the displaced fluid element of the rotating star has the same values of pressure and energy density. In other words, the Lagrangian perturbations of the thermodynamical quantities $\epsilon, P$ vanish [see [27, Eq. (6)]; the modification of these quantities is only due to the displacement (10):

$$
\begin{aligned}
\delta \epsilon(r, \theta) & =-\frac{d \epsilon}{d r} \xi(r, \theta), \\
\delta P(r, \theta) & =-\frac{d P}{d r} \xi(r, \theta) .
\end{aligned}
$$

We remark that as long as we neglect terms of $O\left(\Omega^{4}\right), \delta \epsilon(r, \theta) \simeq \delta \epsilon(\bar{r}, \theta)$.
Einstein's equations, expanded in powers of $\Omega$ and in Legendre polynomials, can be written as a set of ordinary differential equations for the perturbation functions; these equations are summarized in the Appendix. For each value of the central pressure $p_{c}$ (or, equivalently, of the central energy density $\epsilon_{c}$ ) and of the rotation rate $\Omega$, the numerical integration of the perturbation equations yields the perturbed functions, and then the values of the multipole moments of the star (in particular, the mass $M$ and the angular momentum $J$ ) and of its baryonic mass $M_{b}$. These quantities can be written as $M=M^{(0)}+\delta M, J=\delta J$, $M_{b}=M_{b}^{(0)}+\delta M_{b}$, etc., where the quantities with superscript ${ }^{(0)}$ refer to the nonrotating star with central pressure $p_{c}$, and the quantities with $\delta$ are the corrections due to rotation.

Given a nonrotating star with central pressure $p_{c}$ and baryon mass $M_{b}$, the rotating star (with spin $\Omega$ ) with the same central pressure has a baryon mass $M_{b}^{(0)}+\delta M_{b}$, which is generally larger than $M_{b}$. Therefore, a rotating star with same baryon mass $M_{b}$ as the nonrotating one has necessarily a smaller value of the central pressure, $p_{c}+\delta p_{c}$, with $\delta p_{c}<0$ (this is not surprising: when a star is set into rotation, its central pressure decreases).

We mention that in [32] the neutrino transport equations for a rotating star in general relativity have been solved by using an alternative approach. In this approach (which is believed to be accurate for slowly rotating stars [32]) the structure and transport equations for a spherically symmetric star are modified by adding a centrifugal force term to include the effect of rotation.

\section{B. Including the thermodynamical profiles}

In order to integrate the structure equations of a cold neutron star we need to assign an equation of state which, in the case of PNSs, is nonbarotropic, i.e., $\epsilon=\epsilon\left(p, s, Y_{L}\right)$; thus, we also need to know the profiles of entropy and lepton fraction throughout the star. As discussed in Sec. II, these profiles are obtained by our evolutionary code for spherical, nonrotating PNS at selected values of time.

The nonrotating profiles can be used to compute the structure of a rotating PNS in different ways. A possible approach is the following.

Let us consider a spherical PNS with baryon mass $M_{b}$ at a given value of the evolution time $t$. The numerical code discussed in Sec. II provides the functions $p(a), \epsilon(a), s(a)$, $Y_{L}(a)$, where we remind that $a$ is the enclosed baryon number. If we replace the inverse function of $p(a)$ into the nonbarotropic EOS, we obtain an "effective barotropic EOS", $\tilde{\epsilon}(p)=\epsilon\left(p, s(a(p)), Y_{L}(a(p))\right.$, which can be used to solve the TOV equations for the spherical configuration to which we add the perturbations due to rotation, according to Hartle's procedure. Since the rotating star must have the same baryon mass as the spherical star, one can proceed as follows: (i) solve the TOV equations for a spherically 
symmetric star with central pressure $p_{c}+\delta p_{c}$, (ii) solve the perturbation equations for a chosen value of the rotation rate to determine the actual baryon mass of the rotating star with same central pressure, and (iii) iterate these two steps modifying $\delta p_{c}$ until the baryon mass coincides with the assigned value $M_{b}$. This approach was used in [19], where the rotating star was modeled solving the fully nonlinear Einstein equations.

However, this procedure has some relevant drawbacks. Indeed, during the first second after bounce, the star is very weakly bound, and it may happen that the procedure above yields $\delta p_{c}>0$, which indicates that these configurations are in the unstable branch of the mass-radius diagram. We think that this is caused by the unphysical treatment of the thermodynamical profile (effectively, as a barotropic EOS).

This problem did not occur in the simulations of [19] because the authors considered a different, stable branch of the mass-radius curve corresponding to the "effective" EOS $\tilde{\epsilon}(p)$, at much lower densities. Indeed, for $t \lesssim 0.5 \mathrm{~s}$, at the center of the star, they had $n_{b} \sim\left[10^{-2}\right] \mathrm{fm}^{-3}$ (i.e., rest-mass density $\rho \sim\left[10^{13}\right] \mathrm{g} / \mathrm{cm}^{3}$ ), which corresponds to the outer region of the star modeled in [3]. When the central density is so low, only a small region of the star is described by the GM3 EOS; the rest is described by the low-density EOS used to model the PNS envelope, which does not yield unstable configurations.

Since we want to model the PNS consistently with the evolutionary models given in [3], we decided to implement the nonrotating profiles in an alternative way. As in the previous approach, we consider the spherical configuration obtained by the evolution code at time $t$, with central density $p_{c}$ and baryon mass (constant during the evolution) $M_{b}$. To describe the rotating star, we use the GM3 EOS $\epsilon=\epsilon\left(p, s, Y_{L}\right)$; since we are restricting our analysis to slowly rotating stars, the entropy and lepton fraction profiles $s(a)$ and $Y_{L}(a)$ of the nonrotating star are a good approximation for those of the rotating star. We follow the steps discussed at the end of Sec. III A: (i) solve the TOV equations for a star with central pressure $p_{c}+\delta p_{c}$; at each value of $a$, the energy density is $\epsilon\left(p, s(a), Y_{L}(a)\right)$, (ii) solve Hartle's perturbation equations, finding the baryon mass of the star rotating to a given rate with this reduced central pressure and find the correction to the baryon mass due to rotation, and (iii) iterate the first two steps, finding $\delta p_{c}$ such that the baryon mass of the rotating star is $M_{b}$. We remark that the energy density of the rotating star in step (ii) is related to that of the nonrotating star in step (i) by the Hartle-Thorne prescription described above Eq. (11).

Since we are using an appropriate nonbarotropic EOS, the instability discussed above disappears, and the central pressure of the rotating star is, as expected, smaller than that of the nonrotating star with same baryon mass.

We stress again that we are using the numerical solution of the transport equations (5) for a non-rotating PNS to build quasistationary configurations of a rotating PNS.
Therefore, we are neglecting the effect of rotation on the time evolution of the PNS. To be consistent, we should have integrated the transport equations appropriate for a rotating star, which are much more complicated. Since these approximations affect the time scale of the stellar evolution, we would like to estimate how much faster, or slower, the rotating star loses its thermal and lepton content with respect to the nonrotating one. Since the evolution time scale is governed by neutrino diffusion processes, at each time step of the nonrotating PNS evolution, we have computed and compared the neutrino diffusion coefficients $D_{n}$ [see Eqs. (6) and (7)] for nonrotating and rotating configurations. The latter have been obtained by replacing the profiles $(p(a), \epsilon(a)$, etc.) of a nonrotating PNS with those of a rotating PNS (computed as discussed above in this section). In the upper and middle panels of Fig. 3 we plot $D_{2}, D_{3}$, and $D_{4}$ as functions of the enclosed baryon mass $m_{B}=m_{n} a$ for the nonrotating (solid line) and rotating (dashed line) configurations at $t=0.2 \mathrm{~s}, t=$ $1.2 \mathrm{~s}$ and $t=10 \mathrm{~s}$. In the lower panels we plot the neutrino number density and the total energy density at the same times. We assume $M_{b}=1.6 M_{\odot}$ and that the initial angular momentum, $J_{\text {in }}$, is equal to the maximum angular momentum $J_{\max }$, above which mass-shedding sets in (see Sec. IV A for further details). We see that the diffusion coefficients of the rotating configurations are larger than those of the nonrotating star. For $m_{B} \lesssim 1 M_{\odot}$ the relative difference $\left|D_{n}^{\text {rot }}-D_{n}^{\text {non rot }}\right| /\left|D_{n}^{\text {non rot }}\right|$ is always smaller than $\sim 10 \%-20 \%$ and becomes smaller than a few percent after the first few seconds.

In the outer region $m_{B} \gtrsim 1 M_{\odot}$ and early times, the relative difference seems larger, in particular, for the coefficient $D_{3}$, but this has no effect for two reasons. First, as shown in the two lower panels of Fig. 3, both the neutrino number density and the total energy density are much smaller than in the inner core; therefore, even though the diffusion coefficients of the rotating star are larger than those of the nonrotating one, few neutrinos are trapped in this region and transport effects do not contribute significantly to the overall evolution. Second, the differences become large in the semitransparent region, when the mean free path becomes comparable to (or larger than) the distance to the star surface. In this region the diffusion approximation breaks down, and in practice, the diffusion coefficients are always numerically limited (a flux-limiter approach).

From the above discussion, we can conclude that the rotating star loses energy and lepton number through neutrino emission faster than the nonrotating one. This effect is larger at the beginning of the evolution, i.e., for $t \lesssim 2 \mathrm{~s}$, and is of the order of $\sim 10 \%-20 \%$ but becomes negligible at later times. Consequently our rotating star cools down and contracts over a time scale which, initially, is $\sim 10 \%-20 \%$ shorter than that of the corresponding nonrotating configuration. 


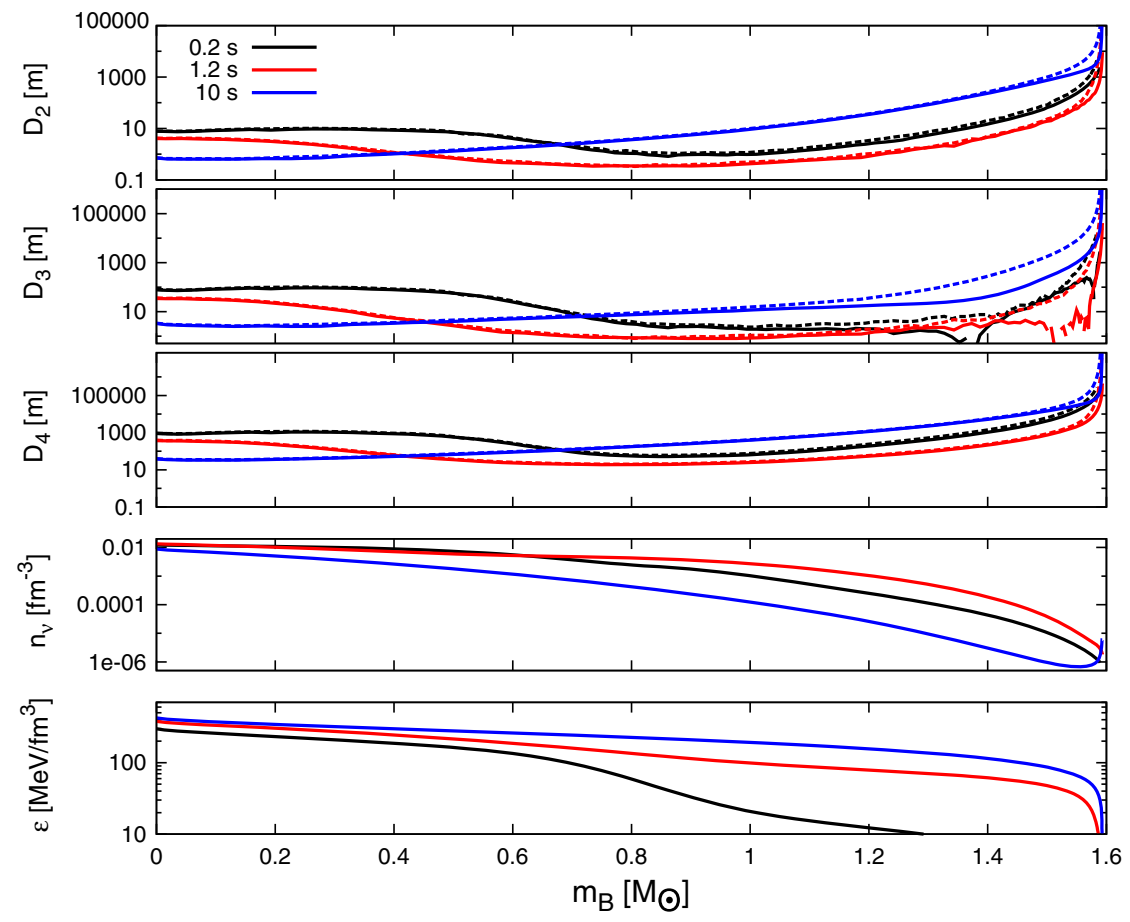

FIG. 3. Neutrino diffusion coefficients $D_{n}(n=2,3,4)$, as functions of the enclosed baryon mass, computed using the density and thermodynamical profiles of the nonrotating (solid line) and rotating (dashed line) configurations at $t=0.2 \mathrm{~s}, t=1.2 \mathrm{~s}$ and $t=10 \mathrm{~s}$ (upper and middle panels). Profiles of neutrino number density and energy density (lower panels). We assume $M_{b}=1.6 M_{\odot}$ and that the angular momentum is the maximum allowed $J_{\text {in }}=J_{\max }$ (for $J_{\text {in }}>J_{\max }$, the PNS reaches the mass-shedding limit during its evolution, see Sec. IVA).

\section{Evolution of the angular momentum and of the rotation rate}

Once the equations describing the rotating configuration are solved for each value of the evolution time $t$ and for an assigned value of the rotation rate $\Omega$, the solution of these equations allows one to compute the multipole moments of the rotating star, including the angular momentum $J$. Conversely we can choose, at each value of $t$, the value of the angular momentum and determine, using a shooting method, the corresponding value of the rotation rate.

If we want to describe the early evolution of a rotating PNS, we need a physical prescription for the time dependence of $J$. For instance, we may assume that the angular momentum is constant, as in [19] (see also [33,34]). However, in the first minute of a PNS life, neutrino emission carries away $\sim 10 \%$ of the star gravitational mass [35] and also a significant fraction of the total angular momentum [36]. To our knowledge, the most sensible estimate of the neutrino angular momentum loss in PNSs has been done by Epstein in [22]

$$
\frac{d J}{d t}=-\frac{2}{5} q L_{\nu} R^{2} \Omega
$$

where $R$ is the radius of the star, $L_{\nu}=-d M / d t$ is the neutrino energy flux, and $q$ is an efficiency parameter, which depends on the features of the neutrino transport and emission. If neutrinos escape without scattering, $q=1$; if, instead, they have a very short mean free path, they are diffused up to the surface and then are emitted with $q=5 / 3$. As discussed in [22] (see also [37-39]), $q=$ $5 / 3$ should be considered as an upper limit of the angular momentum loss by neutrino emission. A more recent, alternative study [40] indicates an angular momentum emission smaller than this limit. In the following, we consider Epstein's formula with $q=5 / 3$, and this has to be meant as an upper limit. We also mention that a simplified expression based on Epstein's formula for the angular momentum loss in PNSs has been derived in [36] and used in [21].

\section{Mass-shedding frequency}

As mentioned in Sec. III A, the perturbative approach which we use to model a rotating star is accurate up to $\nu \lesssim 0.8 \nu_{\mathrm{ms}}$, where $\nu_{\mathrm{ms}}$ is the mass-shedding frequency. The only quantity which is poorly estimated is, of course, the mass-shedding frequency itself. Therefore, $\nu_{\mathrm{ms}}$ is determined using a numerical fit derived in [41] from fully relativistic, nonlinear integrations of Einstein's equations:

$$
\nu_{\mathrm{ms}}(H z)=a \sqrt{\frac{M / M_{\odot}}{R / 1 \mathrm{~km}}}+b,
$$


where $a=45862 \mathrm{~Hz}$ and $b=-189 \mathrm{~Hz}$. We remark that the coefficients $a, b$ of this fit do not depend on the EOS.

\section{E. Gravitational wave emission}

If the evolving PNS is born with some degree of asymmetry, it emits gravitational waves. Assuming that the star rotates about a principal axis of the moment of inertia tensor, i.e., that there is no precession, ${ }^{1}$ gravitational waves are emitted at twice the orbital frequency $\nu$, with amplitude [44-47]

$$
h_{0} \simeq \frac{4 G(2 \pi \nu)^{2} I_{3} \epsilon}{c^{4} r} .
$$

The deviation from axisymmetry is described by the ellipticity $\epsilon$, defined as

$$
\epsilon=\frac{I_{1}-I_{2}}{I_{3}},
$$

where $I_{1}, I_{2}$, and $I_{3}$ are the principal moments of inertia of the PNS and $I_{3}$ is assumed to be aligned with the rotation axis. For old neutron stars, the loss of energy through gravitational waves is compensated by a decrease of rotational energy, which contributes to the spin-down of the star (the main contribution to the spin-down being that of the magnetic field).

In the case of a newly born PNS the situation is different. As the star contracts, due to the processes related to neutrino production and diffusion, its rotation rate increases. If the PNS has a finite ellipticity, it emits gravitational waves, whose amplitude and frequency also increase as the star spins up. The time scale of this process is of the order of tens of seconds. In our model, for simplicity we assume that the PNS ellipticity remains constant over this short time interval.

Unfortunately, the ellipticity of a PNS is unknown. In cold, old NSs it is expected to be, at most, as large as $\sim 10^{-5}-10^{-4}[48,49]$ (larger values are allowed for EOS including exotic matter phases [50,51]). For newly born PNSs, it may be larger, but we have no hint on its actual value. To our knowledge, current numerical simulations of core collapse do not provide estimates of the PNS ellipticity. We remark that although there is observational evidence of large asymmetries in supernova explosions $[52,53]$, there is no evidence that they can be inherited by the PNS. In the following, we assume $\epsilon=10^{-4}$, but this should be considered as a fiducial value: The gravitational wave amplitude (which is linear in $\epsilon$ ) can be easily rescaled for different values of the PNS ellipticity.

\footnotetext{
${ }^{1}$ Free precession requires the existence of a rigid crust [42]; thus, it should not occur in the first tens of seconds of the PNS life, when the crust has not formed yet [43].
}

\section{RESULTS}

\section{A. Spin evolution of the proto-neutron star}

In Fig. 4 we show how the angular momentum changes according to Epstein's formula (12) as the PNS evolves. We assume $q=5 / 3$ and baryonic mass $M_{b}=1.6 M_{\odot}$. We consider different values of the angular momentum $J_{\text {in }}$ at the beginning of the quasistationary phase $(t=0.2 \mathrm{~s}$ after the bounce): $J_{\text {in }}=\left[2.02 \times 10^{48}\right] \mathrm{ergs}, J_{\text {in }}=\left[3.71 \times 10^{48}\right] \mathrm{ergs}$, and $J_{\text {in }}=\left[8.08 \times 10^{48}\right] \mathrm{erg} \mathrm{s}$. We find that, in the first ten seconds after bounce, $13 \%$ of the initial angular momentum is carried away by neutrinos if $J_{\text {in }}=\left[2.02 \times 10^{48}\right] \mathrm{erg}$ s or $J_{\text {in }}=\left[3.71 \times 10^{48}\right] \mathrm{erg} \mathrm{s} ; 20 \%$ of the initial angular momentum is carried away if $J_{\text {in }}=\left[8.075 \times 10^{48}\right] \mathrm{erg}$ s. As mentioned above, $q=5 / 3$ should be considered as an upper bound; for smaller values of $q$, the rate of angular momentum loss would be smaller.

The corresponding evolution of the PNS rotation frequency is shown in Fig. 5. In the same figure we also show the mass-shedding frequency $\nu_{\mathrm{ms}}$, computed using the fit (13). We see that if $J_{\text {in }}=\left[8.08 \times 10^{48}\right] \mathrm{erg} \mathrm{s}$, the curves of $\nu(t)$ and of $\nu_{\mathrm{ms}}(t)$ cross during the quasistationary evolution; before the crossing, the PNS spin is larger than the mass-shedding limit. This means that a PNS with such initial angular momentum would lose mass. If we require the initial rotation rate to be smaller than the mass-shedding limit, we must impose $J_{\text {in }} \leq J_{\max } \equiv\left[3.72 \times 10^{48}\right]$ erg s. We remark that the value of $J_{\max }$ is not affected by the efficiency of angular momentum loss $q$ : If $q<5 / 3, J_{\max }$ has the same value, but the rotation rate grows more rapidly than in Fig. 5.

It is interesting to note that since $\nu_{\mathrm{ms}}$ has a steeper increase than $\nu(t)$, even when the bound $\nu \leq \nu_{\mathrm{ms}}$ is saturated at the beginning of the quasi-stationary phase, the frequency becomes much smaller than the mass

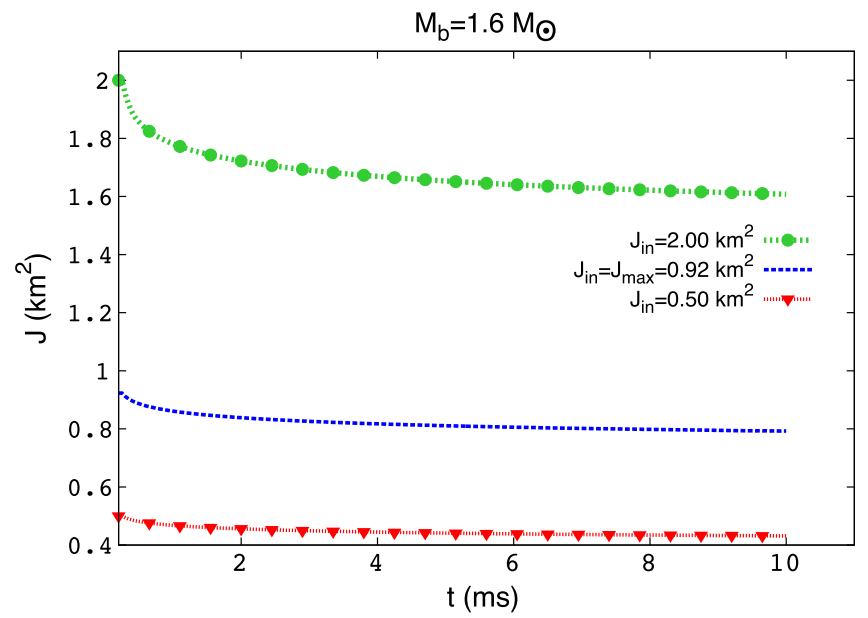

FIG. 4. Angular momentum evolution due to neutrino losses for a PNS with baryonic mass $M_{b}=1.6 M_{\odot}$ and initial angular momentum $J_{\text {in }}=(2.02,3.71,8.08) \times 10^{48} \mathrm{erg} \mathrm{s}$. 
CAMELIO, GUALTIERI, PONS, and FERRARI

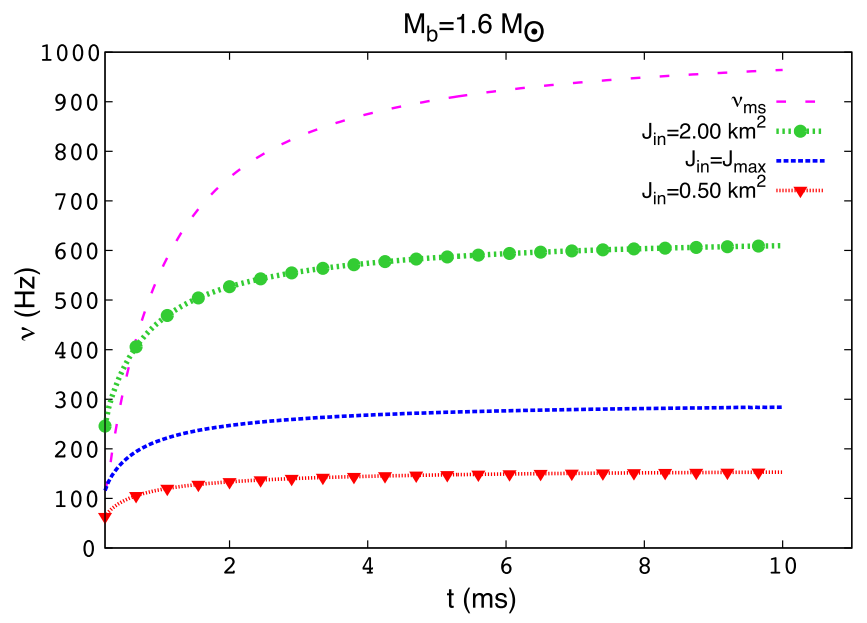

FIG. 5. Evolution of the PNS rotation rate, corresponding to the angular momentum profiles shown in Fig. 4.

shedding frequency at later times. This is an a posteriori confirmation that the slow rotation approximation is appropriate to study newly born PNSs. For $t>10 \mathrm{~s}$, the PNS radius does not change significantly, and the star starts to spin down due to electromagnetic and gravitational emission. However this spin-down time scale is much longer than the time scale of the quasistationary evolution we are considering; therefore it is unlikely that after this early phase the PNS rotation rate is larger than $\sim 300 \mathrm{~Hz}$ (i.e., that its period is smaller than $\sim 3 \mathrm{~ms}$ ), unless some spin-up mechanism (such as, e.g., accretion) sets in. A less efficient angular momentum loss $(q<5 / 3)$ would moderately increase this final value, but the general picture would remain the same.

It is worth noting that models of presupernova stellar evolution [8] predict a similar range of the PNS rotation rate and angular momentum. Among the models considered in [8], the only one with $J>J_{\max }$ (and rotation period smaller than $3 \mathrm{~ms}$ ) is expected to collapse to a black hole. Other works $[9,10]$ have shown that if the progenitor has a rotation rate sufficiently large, the PNS resulting from the core collapse can have periods of a few milliseconds; our results suggest that this scenario is unlikely, unless there is a significant mass loss in the early Kelvin-Helmoltz phase.

\section{B. Gravitational wave emission}

If the PNS has a finite ellipticity $\epsilon$ (which we assume, for simplicity, to remain constant during the first $\sim 10 \mathrm{~s}$ of the PNS life), it emits gravitational waves with frequency $f(t)=2 \nu(t)$ and amplitude given by Eq. (14),

$$
h_{0} \simeq \frac{4 G(2 \pi \nu(t))^{2} I_{3}(t) \epsilon}{c^{4} r} .
$$

As the spin rate $\nu(t)$ increases, both the frequency and the amplitude of the gravitational wave increase; therefore, the signal is a sort of "chirp." This is different from the chirp
PHYSICAL REVIEW D 94, 024008 (2016)

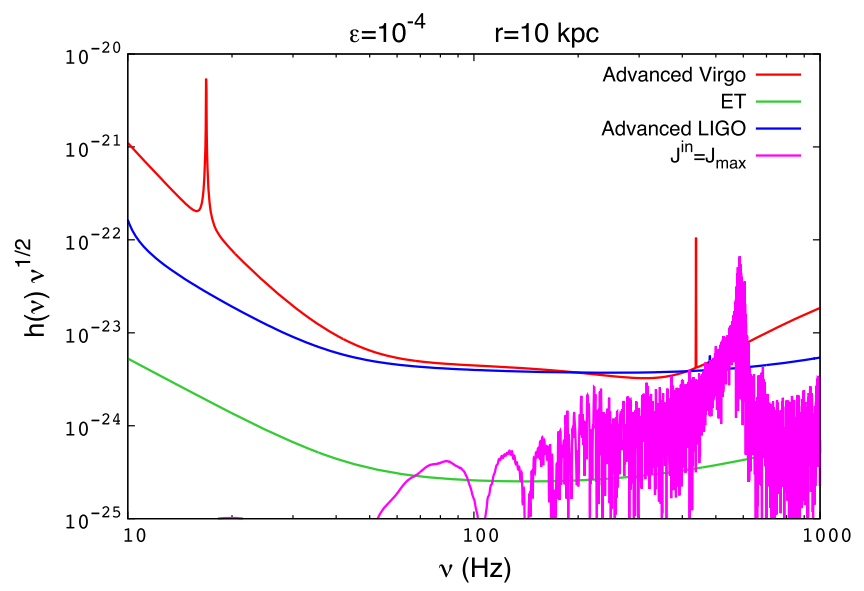

FIG. 6. The strain amplitude $\tilde{h}(f) \sqrt{f}$ of the gravitational wave signal emitted by a PNS with $\epsilon=10^{-4}, J_{\text {in }}=J_{\text {max }}$, at a distance $r=10 \mathrm{kpc}$, is compared with the noise curves of Advanced Virgo, Advanced LIGO, and ET.

emitted by neutron star binaries before coalescence because the amplitude increases at a much milder rate. In Fig. 6 we show the strain amplitude $\tilde{h}(f) \sqrt{f}=$ $\sqrt{f} \sqrt{\left(\tilde{h}_{+}(f)^{2}+\tilde{h}_{\times}(f)^{2}\right) / 2}$, where $\tilde{h}_{+, \times}(f)$ are the Fourier transform of the two polarization of the gravitational wave signal

$$
\begin{gathered}
h_{+}=h_{0} \frac{1+\cos ^{2} i}{2} \cos (2 \pi f(t) t) \\
h_{\times}=h_{0} \cos i \sin (2 \pi f(t) t),
\end{gathered}
$$

and $i$ is the angle between the rotation axis and the line of sight. In Fig. 6 the signal strain amplitude, computed assuming optimal orientation, $J_{\text {in }}=J_{\max }, \epsilon=10^{-4}$ and a distance of $r=10 \mathrm{kpc}$, is compared with the sensitivity curves of Advanced Virgo, ${ }^{2}$ Advanced LIGO, ${ }^{3}$ and of the third generation detector ET. ${ }^{4}$ We see that the signal is marginally above noise for the advanced detectors, but it is definitely above the noise curve for ET. This signal would be seen by Advanced Virgo with a signal-to-noise ratio $\mathrm{SNR}=1.4$ and by Advanced LIGO with $\mathrm{SNR}=2.2$, too low to extract it from the detector noise; however, since the signal-to-noise ratio scales linearly with the ellipticity, a star born with $\epsilon=10^{-3}$ would be detected with SNR $=14$ and SNR $=22$ by Advanced Virgo and LIGO, respectively. The third generation detectors like ET would detect the signal coming from a galactic PNS born with $\epsilon=10^{-4}$ with a very large signal-to-noise ratio, i.e., $\mathrm{SNR}=22$. If the source is in the Virgo cluster $(d=15 \mathrm{Mpc})$, the ellipticity

\footnotetext{
${ }^{2}$ https://inspirehep.net/record/889763/plots

${ }^{3}$ https://dcc.ligo.org/LIGO-T0900288/public

${ }^{4}$ http://www.et-gw.eu/etsensitivities
} 
of the PNS should be as large as $5 \times 10^{-2}$ to be seen by ET with $\mathrm{SNR}=8$.

\section{CONCLUDING REMARKS}

In this paper we have studied the angular momentum loss, the time dependence of the rotation rate, and the gravitational wave emission of a newly born PNS during the first tens of seconds after bounce. The early evolution of the rotating PNS has been modeled using the entropy and lepton fraction profiles consistently computed solving the general relativistic transport equations for a nonrotating star; angular momentum loss due to neutrino emission has been modeled using Epstein's formula [22].

During this early evolution, the star spins up due to contraction. By requiring that the initial rotation rate does not exceed the mass-shedding limit, we have estimated the maximum rotation rate at the end of the spin-up phase. For a PNS of $M_{b}=1.6 M_{\odot}$, we find that one minute after bounce the star would rotate at $\nu \lesssim 300 \mathrm{~Hz}$, corresponding to a rotation period $\tau_{\min } \gtrsim\left[3.3 \times 10^{-3}\right] \mathrm{s}$.

If the PNS is born with a finite ellipticity $\epsilon$, while spinning up, it emits gravitational waves at twice the rotation frequency. This signal increases both in frequency and amplitude. We find that for a galactic supernova, if $\epsilon=10^{-3}$, this signal could be detected by Advanced LIGO/Virgo with a signal-to-noise ratio $\gtrsim 14$. To detect farther sources, third generation detectors like ET would be needed.

We remark that the actual value of PNS ellipticities is unknown and depends on the details of the supernova core collapse. Accurate numerical simulations of supernova explosion addressing this issue are certainly needed to provide a quantitative estimate of the range of $\epsilon$.

We also remark that in our approach the effects of the PNS rotation are consistently included in the structure equations, but they are neglected when solving the neutrino transport equations. We estimate that due to this approximation, we overestimate the evolution time scale at early times of, at most, $\sim 10 \%-20 \%$. Moreover, since we are not interested in the details of the neutrino dynamics and we need a fast code to evolve the star for tens of seconds, we perform energy averages to determine the neutrino diffusion coefficients, and we apply a flux-limiter. These approximations should not significantly affect the thermodynamical evolution of the PNS and its gravitational wave emission.

This work is a first step in the study of the early evolution of PNSs. A paper with a detailed description of our numerical code, and its extension to more recent EOSs, is in preparation [23]. Further developments shall include differential rotation, convection, and generalization of the neutrino transport equations to rotating PNSs.

\section{ACKNOWLEDGMENTS}

We thank O. Benhar and A. Lovato for useful discussions on the EOS of the PNS. We also thank S. Reddy and
L. F. Roberts for useful discussions on the neutrino cross sections. This work was partially supported by "NewCompStar" (COST Action MP1304), and by the H2020-MSCA-RISE-2015 Grant No. StronGrHEP-690904. J.A.P. acknowledges support by the MINECO Grants No. AYA2013-42184-P and No. AYA2015-66899-C2-2-P.

\section{APPENDIX: HARTLE-THORNE EQUATIONS}

Here, we briefly describe the equations of the perturbative Hartle-Thorne approach discussed in Sec. III A. For further details, we refer the reader to $[27,28,54]$ and to the Appendix of [29].

The spacetime metric [up to order $O\left(\Omega^{3}\right)$ ] is given by Eq. (8); it depends on the background functions $\phi(r)$, $\lambda(r)=-\log (1-2 m(r) / r)$, and on the perturbations functions $h_{l}(r), m_{l}(r)(l=0,2), k_{2}(r), \omega(r), w_{l}(r)(l=1,3)$. The energy and pressure (Eulerian) perturbations are

$$
\begin{aligned}
\delta P & =(\epsilon(r)+P(r))\left(\delta p_{0}(r)+\delta p_{2}(r) P_{2}(\mu)\right) \\
\delta \epsilon & =\frac{d \epsilon / d r}{d P / d r} \delta P
\end{aligned}
$$

and depend on the perturbation functions $p_{l}(r)(l=0,2)$.

The background spacetime is described by the TOV equations:

$$
\begin{aligned}
& \frac{d m}{d r}=4 \pi r^{2} \epsilon \\
& \frac{d \phi}{d r}=2 \frac{m+4 \pi r^{3} P}{r(r-2 m)} \\
& \frac{d P}{d r}=-\frac{\epsilon+P}{2} \frac{d \phi}{d r} .
\end{aligned}
$$

The mass of the nonrotating configuration is obtained by matching at the stellar surface $r=R$ the interior solution with the exterior (Schwarzschild) solution, i.e., $M=m(R)$. Moreover, the baryonic mass $M_{b}$ of the nonrotating configuration is obtained integrating the equation $d m_{b} / d r=4 \pi r^{2} e^{\lambda / 2} \rho$ and computing $M_{b}=m_{b}(r)$.

The spacetime perturbation to first order in $\Omega$ is described by the function $\omega(r)$, which is responsible for the dragging of inertial frames; it satisfies the equations

$$
\begin{gathered}
\frac{d \chi}{d r}=\frac{u}{r^{4}}-\frac{4 \pi r^{2}(\epsilon+P) \chi}{r-2 M} \\
\frac{d u}{d r}=\frac{16 \pi r^{5}(\epsilon+P) \chi}{r-2 M},
\end{gathered}
$$

where $\varpi=\Omega-\omega, j(r)=e^{-\phi / 2}(1-2 M / r)^{1 / 2}, \chi=j \varpi$, and $u=r^{4} j d \varpi / d r$. The angular momentum $J$ is obtained by matching the interior with the exterior solution $\chi(r)=\Omega-2 J / r^{3}, u(r)=6 J$ at $r=R$. The moment of inertia, at zeroth order in the rotation rate, is $I=J / \Omega$. 
The perturbations to second order in $\Omega$ are described by the metric functions $h_{l}(r), m_{l}(r)(l=0,2), k_{2}(r)$, and by the fluid pressure perturbations $\delta p_{l}$. The $l=0$ perturbations satisfy the equations

$$
\begin{aligned}
\frac{d}{d r}\left(\delta p_{0}+h_{0}-\frac{\chi^{2} r^{3}}{3(r-2 M)}\right) & =0 \\
\delta p_{2}+h_{2}-\frac{\chi^{2} r^{3}}{3(r-2 M)} & =0
\end{aligned}
$$

and

$$
\begin{aligned}
\frac{d m_{0}}{d r} & =4 \pi r^{2} \frac{d \epsilon}{d P}\left[\delta p_{0}(\epsilon+P)\right]+\frac{u^{2}}{12 r^{4}}+\frac{8 \pi r^{5}(\epsilon+P) \chi^{2}}{3(r-2 M)} \\
\frac{d \delta p_{0}}{d r} & =\frac{u^{2}}{12 r^{4}(r-2 M)}-\frac{m_{0}\left(1+8 \pi r^{2} P\right)}{(r-2 M)^{2}}-\frac{4 \pi(\epsilon+P) r^{2} \delta p_{0}}{r-2 M}+\frac{2 r^{2} \chi}{3(r-2 M)}\left[\frac{u}{r^{3}}+\frac{\left(r-3 M-4 \pi r^{3} P\right) \chi}{r-2 M}\right] .
\end{aligned}
$$

Matching the interior and the exterior solutions at $r=R$, it is possible to compute the correction to the mass due to stellar rotation, $\delta M=m_{0}(R)+J^{2} / R^{3}$, and the monopolar stellar deformation. The baryonic mass correction $\delta M_{b}=\delta m_{b}(R)$ is given by solving the equation

$$
\frac{d \delta m_{b}}{d r}=4 \pi r^{2} e^{\lambda / 2}\left[\left(1+\frac{m_{0}}{r-2 m}+\frac{1}{3} r^{2} \varpi^{2} e^{-\phi}\right) \epsilon+\frac{d \epsilon / d r}{d P / d r}(\epsilon+P) \delta p_{0}\right] .
$$

The $l=2$ perturbations satisfy the equations

$$
\begin{aligned}
\frac{d v_{2}}{d r}= & -\frac{d \phi}{d r} h_{2}+\left(\frac{1}{r}+\frac{1}{2} \frac{d \phi}{d r}\right)\left[\frac{8 \pi r^{5}(\epsilon+P) \chi^{2}}{3(r-2 M)}+\frac{u^{2}}{6 r^{4}}\right] \\
\frac{d h_{2}}{d r}= & {\left[-\frac{d \phi}{d r}+\frac{r}{r-2 M}\left(\frac{d \phi}{d r}\right)^{-1}\left(8 \pi(\epsilon+P)-\frac{4 M}{r^{3}}\right)\right] h_{2}-\frac{4 v_{2}}{r(r-2 M)}\left(\frac{d \phi}{d r}\right)^{-1}+\frac{u^{2}}{6 r^{5}}\left[\frac{1}{2} \frac{d \phi}{d r} r-\frac{1}{r-2 M}\left(\frac{d \phi}{d r}\right)^{-1}\right] } \\
& +\frac{8 \pi r^{5}(\epsilon+P) \chi^{2}}{3(r-2 M)}\left[\frac{1}{2} \frac{d \phi}{d r} r+\frac{1}{r-2 M}\left(\frac{d \phi}{d r}\right)^{-1}\right],
\end{aligned}
$$

where $v_{2}=k_{2}+h_{2}$. Matching the interior and exterior solutions, it is possible to determine the quadrupole moment of the PNS and its quadrupolar deformation.

The equations for the peturbations at $O\left(\Omega^{3}\right), w_{l}(r)(l=1,3)$, have a similar structure, but they are longer and are not reported here. We refer the reader to $[29,54]$. They yield the octupole moment, the third-order corrections to the angular momentum, and the second-order corrections to the moment of inertia.

[1] A. Burrows and J. M. Lattimer, Astrophys. J. 307, 178 (1986).

[2] W. Keil and H.-T. Janka, Astron. Astrophys. 296, 145 (1995).

[3] J. A. Pons, S. Reddy, M. Prakash, J. M. Lattimer, and J. A. Miralles, Astrophys. J. 513, 780 (1999).

[4] V. Ferrari, G. Miniutti, and J. A. Pons, Mon. Not. R. Astron. Soc. 342, 629 (2003).

[5] C.D. Ott, Classical Quantum Gravity 26, 063001 (2009).
[6] G. F. Burgio, V. Ferrari, L. Gualtieri, and H. J. Schulze, Phys. Rev. D 84, 044017 (2011).

[7] J. Fuller, H. Klion, E. Abdikamalov, and C. D. Ott, Mon. Not. R. Astron. Soc. 450, 414 (2015).

[8] A. Heger, S. E. Woosley, and H. C. Spruit, Astrophys. J. 626, 350 (2005).

[9] T. A. Thompson, E. Quataert, and A. Burrows, Astrophys. J. 620, 861 (2005).

[10] C. D. Ott, A. Burrows, T. A. Thompson, E. Livne, and R. Walder, Astrophys. J. Suppl. Ser. 164, 130 (2006). 
[11] F. Hanke, B. Mueller, A. Wongwathanarat, A. Marek, and H.-T. Janka, Astrophys. J. 770, 66 (2013).

[12] S. M. Couch and C. D. Ott, Astrophys. J. 799, 5 (2015).

[13] K. Nakamura, T. Kuroda, T. Takiwaki, and K. Kotake, Astrophys. J. 793, 45 (2014).

[14] M. C. Miller and J. M. Miller, Phys. Rep. 548, 1 (2015).

[15] J. A. Pons, J. A. Miralles, M. Prakash, and J. M. Lattimer, Astrophys. J. 553, 382 (2001).

[16] J. A. Pons, A. W. Steiner, M. Prakash, and J. M. Lattimer, Phys. Rev. Lett. 86, 5223 (2001).

[17] L. F. Roberts, Astrophys. J. 755, 126 (2012).

[18] V. Ferrari, L. Gualtieri, J. A. Pons, and A. Stavridis, Mon. Not. R. Astron. Soc. 350, 763 (2004).

[19] L. Villain, J. A. Pons, P. Cerda-Duran, and E. Gourgoulhon, Astron. Astrophys. 418, 283 (2004).

[20] E. Gourgoulhon, P. Haensel, R. Livine, E. Paluch, S. Bonazzola, and J.-A. Marck, Astron. Astrophys. 349, 851 (1999).

[21] G. Martinon, A. Maselli, L. Gualtieri, and V. Ferrari, Phys. Rev. D 90, 064026 (2014).

[22] R. Epstein, Astrophys. J. 219, L39 (1978).

[23] G. Camelio, L. Gualtieri, G. Lovato, O. Benhar, J. A. Pons, M. Fortin, and V. Ferrari (to be published).

[24] N. K. Glendenning and S. A. Moszkowski, Phys. Rev. Lett. 67, 2414 (1991).

[25] C. Levermore and G. Pomraning, Astrophys. J. 248, 321 (1981).

[26] J. R. Wilson and R. W. Mayle, in NATO Advanced Science Institutes (ASI), Series B, Vol. 216, edited by W. Greiner and H. Stöcker (Springer, New York, 1989) p. 731.

[27] J. B. Hartle, Astrophys. J. 150, 1005 (1967).

[28] J. B. Hartle and K. S. Thorne, Astrophys. J. 153, 807 (1968).

[29] O. Benhar, V. Ferrari, L. Gualtieri, and S. Marassi, Phys. Rev. D 72, 044028 (2005).

[30] H.-T. Janka and R. Mönchmeyer, Astron. Astrophys. 226, 69 (1989).

[31] P. Mösta, C. D. Ott, D. Radice, L. F. Roberts, E. Schnetter, and R. Haas, Nature (London) 528, 376 (2015).

[32] E. O'Connor and C. D. Ott, Classical Quantum Gravity 27, 114103 (2010).
[33] J. O. Goussard, P. Haensel, and J. L. Zdunik, Astron. Astrophys. 321, 822 (1997).

[34] J. O. Goussard, P. Haensel, and J. L. Zdunik, Astron. Astrophys. 330, 1005 (1998).

[35] J. M. Lattimer and M. Prakash, Astrophys. J. 550, 426 (2001).

[36] H.-T. Janka, IAU Symposium 218: Young Neutron Stars and Their Environment, Sydney, Australia, July 14-17, 2003, (2004).

[37] D. Kazanas, Nature (London) 267, 501 (1977).

[38] K. O. Mikaelian, Astrophys. J. 214, L23 (1977).

[39] R. N. Henriksen and W. Y. Chau, Astrophys. J. 225, 712 (1978).

[40] M. Dvornikov and C. Dib, Phys. Rev. D 82, 043006 (2010).

[41] D. D. Doneva, E. Gaertig, K. D. Kokkotas, and C. Krger, Phys. Rev. D 88, 044052 (2013).

[42] D. I. Jones and N. Andersson, Mon. Not. R. Astron. Soc. 324, 811 (2001).

[43] Y. Suwa, Publ. Astron. Soc. Jpn. 66, L1 (2014).

[44] M. Zimmermann and E. Szedenits, Phys. Rev. D 20, 351 (1979).

[45] K. S. Thorne, in Three Hundred Years of Gravitation, edited by $\mathrm{S}$. W. Hawking and W. Israel (Cambridge University Press, Cambridge, England, 1987), pp. 330-458.

[46] S. Bonazzola and E. Gourgoulhon, Astron. Astrophys. 312, 675 (1996).

[47] D. I. Jones, Classical Quantum Gravity 19, 1255 (2002).

[48] B. Haskell, D. I. Jones, and N. Andersson, Mon. Not. R. Astron. Soc. 373, 1423 (2006).

[49] R. Ciolfi, V. Ferrari, and L. Gualtieri, Mon. Not. R. Astron. Soc. 406, 2540 (2010).

[50] C. J. Horowitz and K. Kadau, Phys. Rev. Lett. 102, 191102 (2009).

[51] N. K. Johnson-McDaniel and B. J. Owen, Phys. Rev. D 88, 044004 (2013).

[52] L. Wang, D. Baade, P. Hoeflich, J. C. Wheeler, C. Fransson, and P. Lundqvist, Astrophys. J. 592, 457 (2003).

[53] D. C. Leonard et al., Nature (London) 440, 505 (2006).

[54] J. B. Hartle, Astrophys. Space Sci. 24, 385 (1973). 